
APPLICATIONS

\author{
C. M. FUNLER, M-6 \\ 8.1171104i::- \\ 1.. IREHMN, $M-6$ \\ . S. BROOKS, BOSTON UNIVERSITY \\ i. D. COETTEE, BOSTON UNIVERSITY \\ ‥ L. HULTS, CM-STC \\ I. C. KING, M-6 \\ Y. MULLLER, CH-CMS \\ i. (i. RTCRl: $M-6$
}

.. 1. SMITH, CM-STC

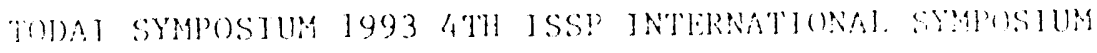

FRONTIERS IN HIGH MAGNETIC FIELDS

NOVEMBER $10-12,1993$

TOKYO, JAPAN

\title{
DISCLAIMER
}

This report was prepared as an account of work sponsored by an agency of the United States Government. Neither the United States Government nor any agency thereof, nor any of their employees, makes any warranty, express or implied, or assumes any legal liability or responsibility for the accuracy, completeness, or usefulness of any information, apparatus, product, or process disclosed, or represents that its use would not infringe privately owned rights. Reference herein to any specific commercial product, process, or service by trade name, trademark, manufacturer, or otherwise does not necessarily constitute or imply its endorsement, recommendation, or favoring by the United States Government or any agency thereof. The views and opinions of authors expressed herein do not necessarily state or reflect those of the United States Government or any agency thereof.

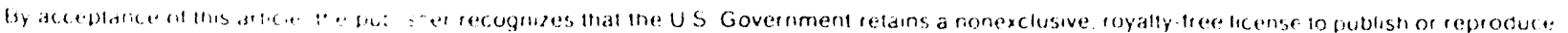

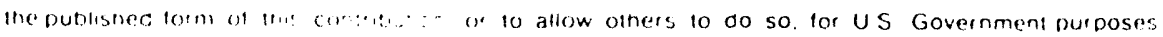

The los Alamos Nationial tacorato. equests that the publistief identify this arficle as work pertormed under ine ausoices of the U $S$ Department of Energ,

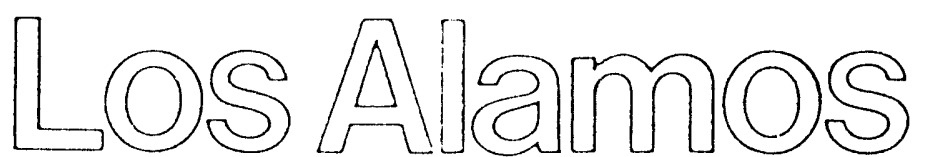




\section{EXPI,OSIVELY PRODUCED MEGAGAUSS FIELDS AND RECENT SOLID STATE APPLICATIONS}

C. M. Fowler, ${ }^{a}$ B. L. Freeman,${ }^{a}$ J. S. Brooks, ${ }^{b}$ J. D. Gocttee, ${ }^{b}$ W. L. Hults,,${ }^{a}$ J. C. King,, , F. M. Mueller, ${ }^{a}$ D. G. Rickel ${ }^{a}$ and J. L. Smith ${ }^{a}$

"Los Alamos National Laboratory, Los Alamos, NM 87545, USA.

${ }^{b}$ Department of Physics, Boston University, Boston, MA 02215, USA.

Large magnetic fields may be generated by compression of an initial magnetic flux generated over a large area at relatively low magnetic field into a region of smaller area. Following a discussion of flux compression principles, we discuss megagauss field systems in use at Los Alamos where chemical explosives are used to compress the flux. Their use in some solid state experiments will be discussed briefly, including a planned set of experiments on YBCO to be done jointly with a Russian team, whose aim is to determine the low temperature, critical magnetic ficld of YBC.O.

Keywords: Megagauss fields; explosive flux compression, high temperature superconductors, critical magnetic ficld. IBCO, magneto-resistance.

Correspondence to: C. M. Fowler, MS C970, Los Alamos National Laboratory, Los Alamos, NM S7545, USA.

Phone: (505)-667-6S\&1: FAX: (505)-665-3050 


\section{INTRODUCTION}

Explosive flux compression devices are designed to produce very large magnetic fields or to generate large bursts of energy [1-3]. These devices consist basically of a cavity defined by surrounding metal conductors, some or all of which may be overlaid with explosives. After an initial magnetic flux is introduced into the cavity, the explosives are detonated. The subsequent motion of the conductors leads to compression of the flux into regions of smaller cross-section. Unless flux losses into or through the metal conductors are excessive, magnetic fields and currents are increased as the initial flux is compressed.

The flux compression process may be seen readily from Fig. 1, which is a schematic drawing of our most commonly used high-field system, commonly referred to as the "1MG" strip generator. Initial flux is developed in the triangular generator cavity, bounded by copper sheet, and the cylindrical load coil, drilled out of a brass block. The flux is obtained from capacitor-driven current supplied through the opening at the top left. Strips of sheet explosive mounted on both plates of the generator are detonated near peak system flux. Motion of the top strip first closes the current input slot, thus trapping the flux in the generator volume and load coil. As detonation proceeds along the strips, the generator area is reduced and the current and magnetic field increase in the system. The configuration of the copper plates during compression is shown by dashed lines at a time when the detonation fronts have progressed to the points labelled D.

In the standard configuration, the copper plates are about $300-\mathrm{mm}$ long and $100-\mathrm{mm}$ wide. The explosive strips, about $90-\mathrm{mm}$ wide with total weight of about $2 \mathrm{~kg}$, are cut from commercially available rolls of explosive. The load coils are made from $50.8-\mathrm{mm}$ square brass bar stock. Peak magnetic fields developed in the load coils depend upon the load coil diameter. Typical peak values are $110 \mathrm{~T}$ for $19-\mathrm{mm}$ diameter load coils and $150 \mathrm{~T}$ for 12 -mm diameters. The most commonly used coil length is $76.2 \mathrm{~mm}$, although the peak 
fields developed a: relatively insensitive to the coil length in the range 60 to $100 \mathrm{~mm}$.

Figure 2 shows the magnetic field $B$ and its time derivative $\dot{B}$ as a function of time for a load coil of 12.6- $\mathrm{mm}$ diameter and 76.2-mm long. The initial magnetic field, supplied by a capacitor bank, is approximately $10 \mathrm{~T}$. The explosive detonation is timed so that the generator current input slot is closed at the point marked "a." The maximum value of $d B / d t$, at time $b$, is $1.35 \cdot 10^{7} \mathrm{~T} / \mathrm{s}$. It occurs a few microseconds before the magnetic field peak, $147 \mathrm{~T}$ (time $c$ ). The magnetic probe is destroyed at time $d$ from the shock wave developed by the explosive.

For many situations the elements of a system, such as in Fig. 1, can be described by an inductance $L$ such that the flux and magnetic energy of the element can be written as $L I$ and $1 / 2 L I^{2}$, where $I$ is the total current flowing through the system. Such systems can then be represented by the circuit diagram of Fig. 3. The identification of the circuit elements with the strip generator components of Fig. 1 is as follows. The variable inductor represents the triangular wedge overlaid with sheet explosive while the fixed inductor $L_{1}$, represents the cylindrical load coil. The source inductance term $\ell$ would account for the small transmission line inductance linking the section components as well as the inductance associated with flux penetration into the conductor skins. This latter term is, of course, time dependent. For purposes of illustration we will, however, treat it as constant. The voltage equation for the circuit then becomes:

$$
\frac{d}{d t}(L I)+I R+\ell \frac{d I}{d t}+L_{1} \frac{d I}{d t}=0
$$

or

$$
(\dot{L}+R) I+L \frac{d I}{d t}+\ell \frac{d I}{d t}+L_{1} \frac{d I}{d t}=0
$$

The term $\dot{L}$ is negative since the explosive forcibly reduces the generator inductance. Its value is usually called the generator impedance. It is clear from Eq. (2) that on the average the generator impedance must exceed the resistance $R$ if the current is to increase. 
The voltage term $\dot{L} I$ drives the rest of the circuit whose terms, from Eq. (2), consist of the voltage drop across the resistor, the inductance left in the generator, the source inductance, and the load inductance. This term is equivalent to the motional term, $B \ell v$ for a conductor of length $\ell$ moving through a magnetic field $B$ with velocity $v$.

If the circuit is a perfect conductor, $R=0$, and Eq. (1) then states that the circuit flux is constant.

$$
\left(L(o)+\ell+L_{1}\right) I_{o}=\left(L(t)+\ell+L_{1}\right) I \quad .
$$

Peak current is obtained when the explosive has reduced the generator inductance to zero.

$$
I=\frac{L(o)+\ell+L_{1}}{\ell+L_{1}} I_{o}
$$

For the strip generator of Fig. 1, $L(o)$ is of order $100 \mathrm{nH}$, while the load inductance is typically of order a few $\mathrm{nH}$ for a diameter of say $15 \mathrm{~mm}$ and length $76 \mathrm{~mm}$. With constant diameter, and ignoring edge effects, the load inductance varies inversely with the coil length. When the source inductance is negligible it is then seen from Eq. (4) that the peak current varies approximately as the coil length. This explains why the peak fields do not vary substantially with the coil length, since the peak current per coil length is about the same. However, when the coil length gets too large, the influence of source inductance is greater and the peak fields will decrease with length.

Almost any method of moving a conductor fast enough can, in principle, serve as the basis for a flux compression generator. A number of energy sources have been suggested, including compressed air, explosive gas mixes, and moving machinery - some quite exotic. A number of the devices are described in [4]. Most of these devices have not been used to make large magnetic fields. The exception is the use of outer magnetic fields to compress 
another magnetic field contained within a cylindrical conductor. This technique has been extensively developed by N. Miura and his collaborators [5]. Miura and J.J.M. Franse, in a very recent article [6], discuss fields as high as $550 \mathrm{~T}$ produced in this manner.

\subsection{Generator Losses}

Two basic causes of flux loss are by diffusion of magnetic fields into the conductors and by flux pocketing. To these may be added the leakage of flux through the conductors, which disappears as radiation. This latter term is usually very small unless the thickness of the conductors is comparable to, or smaller than the field diffusion cepth during the operation time of the generators. When the conductors are thin enough, flux flows freely through them. In this case, these conductors may be represented by a resistance, such as that appearing in Eqs. (1) and (2). Such conditions are normally avoided in well-designed, flux-compression systems.

Diffusion losses, while inevitable, are reduced by using better conducting materials, by reducing generator operation time when practicable, and by using wider conductors up to the specified load coil when possible. Wider conductors allow for lower current density and thus lower magnetic fields at the conductor surfaces, with a consequent reduced flux loss. Good discussions of diffusion are given in [1,2], while the problem of flux leakage through the conductors is discussed in [7].

Flux pocketing occurs when part of the flux gets shorted out and isolated from the load. An extreme example can be considered by referring to Fig. 1. If the angle between the moving conductors (intersection of dotted lines) is too large, a metallic jet can form at this junction. The jet will move ahead of the junction and short the input slot to the load coil. The flux remaining between the moving plate junction and the input slot is thus "pocketed" out or lost to the load. Good generator design calls for avoiding such pocketing. 


\subsection{Component Motion From Magnetic Forces}

The generator conductors that compress the flux are themselves subject to Lorenty forces that tend to move them apart. These forces are generally overcome by those generated by the explosive. However, reference to Figs. 1 and 2 show that parts of the conductors ahead of the detonation front can be subjected to large Lorentz forces without benefit of compensation from the explosive pressures. For good performance, allowance must be made for these usually undesirable motions. In the present case, the mass of the copper conductors, together with that of the explosives, limits this motion, at least to the field levels cited and with the field pulse shapes normally encountered. This subject is treated in greater detail in Ref. 8 , from which the following is extracted. Consider the motion of one of the generator plates of Fig. 1. Let $M$ be the mass density $\left(\mathrm{kg} / \mathrm{m}^{2}\right)$ of the plate and explosive and $B(t)$ be the magnetic field in the cavity that exerts pressure on the plate, tending to push it outward. With neglect of edge effects and material strength, the equation of motion of the plate is

$$
M a=B(t)^{2} / 2 \mu
$$

We consider the following pulse shape for $B(t)$ :

$$
B(t)=B_{M}(t / T)^{n}
$$

Here, the magnetic field rises to a maximum value $B_{M}$ in time $T$ with an $n$th power dependency on time. A value of $n=1 / 2$ gives a reasonable representation of a capacitor discharge into an inductor with quarter period $T$. From Fig. 2 , it is seen that a value of $n$ of order two represents fairly well the field dependence from 30 to $70 \mu \mathrm{s}$ with $B_{M}=147 \mathrm{~T}$. Integration of $\mathrm{Eq}$. (5) gives for the peak displacement, $d$, to time $T$

$$
d=\frac{B_{M}^{2} T^{2}}{2 \mu M[(2 n+1)(2 n+2)]}
$$


It is seen from Eq. (7) that the displacement in time $T$ would be five times greater had the same field becn produced by a capacitor discharge. A similar rough analysis shows that joule heating of the plates is also reduced for this kind of time dependence.

One more effect of the pulse shape should be noted. As seen from Fig. 2 when the inductive voltages are large, so are the magnetic fields. This feature affords some protection against electrical breakdown since the high magnetic fields inhibit transfer of charge between the current-carrying conductors (such as the coil feed slot of Fig. 1). This may be contrasted with the typical $L C$ capacitor discharge where voltages are maximum at zero current and field.

\subsection{Magnetic Field Measurements}

From Fig. 2 the magnetic field pulse length is about $6 \mu$ s from $90 \%$ of peak-to-peak field. This value is typical for fields in the 100 to $200-\mathrm{T}$ range, although the time varies with coil material and size and peak-field amplitude. As noted earlier, the time derivative of the magnetic field reaches a peak of $1.35 \times 10^{7} \mathrm{~T} / \mathrm{s}$, a few microseconds before peak field.

Magnetic fields are normally measured by use of an induction probe of known area $A$, normal to the magnetic field. The voltage signal developed around the probe area is $A d B / d t$. The signal is often both electronically integrated during the experiment and numerically integrated later. The signals of Fig. 2 were obtained from calibrated probes consisting of aboút 30 turns of fine wire wound on a 1-mm nylon rod. Areas were typically $30 \mathrm{~mm}^{2}$. From Fig. 2, it is seen that each square millimeter developed a peak voltage of $13.5 \mathrm{~V}$. The total probe voltage was about $400 \mathrm{~V}$. This voltage is quite manageable with the coaxial cables and their fittings used to carry the probe signals. 
The situation is different, however, for the multimegagauss fields produced by liner implosions. For example, $d B / d t$ is of order $6 \times 10^{8} \mathrm{~T} / \mathrm{s}$ for the $550-\mathrm{T}$ field discussed by Franse and Miura [6], and shown plotted on their Fig. 5. This means that voltages as high as $600 \mathrm{~V}$ per $\mathrm{mm}^{2}$ would be developed around the probe. For some of the fields produced by explosive liner implosion, $d B / d t$ values of $2 \times 10^{9} \mathrm{~T} / \mathrm{s}$ have been produced. With resulting peak voltages of $2000 \mathrm{~V} / \mathrm{mm}^{2}$, it is clear that probes of only a few square millimeters would be manageable. Such smaller probe areas normally result in less accurate probe calibrations.

Pavlovskii and his coworkers [9] use Faraday rotation as a field measurement tool. Here, plane-polarized light from a He-Ne laser is directed through a small cylinder of heavy flint glass, with an additional plane polarizer placed between the glass cylinder and the signal detector. Measurement of the amount of rotation of the light is obtained from the transmitted signal, which shows alternate peaks and zeros as the rotation advances in $90^{\circ}$ steps. The magnetic field is then obtained as a function of time by dividing the rotation angle by the glass cylinder length and the appropriate Verdet constant. Comparison of field values obtained this way, with values obtained from inductance probes, shows that the rotation of light of this wavelength remains linear with fields up to $1500 \mathrm{~T}$, at least within the stated accuracy of 2-3\%. If different materials or light wavelengths are used, similar checks for linearity should be made. As noted by Fowler et al. [10] non-linearities can be found in megagauss fields, particularly at shorter wavelengths.

One other field measurement of a more or less absolute type may be mentioned. Garn and his coworkers [11] have measured Zeeman splitting (of well understood transitions) with use of a time-resolved spectrographic camera. Good agreement with probe measurements was obtained up to several hundred Teslas. However, it should be mentioned that the useful field volume was taken up by the Zeeman source, leaving no room for other experiments. 
In summary, inductive probes can be used with great confidence in non-implosion geometries, essentially of fixed volume, such as in the configuration of Fig. 1. For implosionproduced fields, increasing care must be taken with induction probes to avoid voltagebreakdown problems. Here, more reliance on Faraday rotation field measurements may be expected, as larger fields are produced.

\section{EXPERIMENTAL DIAGNOSTICS}

Most of the diagnostics used with these large fields involve either electrical or optical measurements.

\subsection{Optical Diagnostics}

Studies have been done with both single wave-length and broad band light sources. Fowler et al. [12] surveyed the optical studies done in the Los Alamos program until its termination in 1980. Light sources included mercury lamps with the blue and green lines as single wave-length sources and progressed to wide band obtained by shocking argon gas, in a small pipe, with an explosive detonator. The white light from the argon flash, after passing through the sample under investigation, was time and wave-length resolved by a rotating mirror, spectrographic camera, and recorded on photographic film. Investigations included Faraday rotation and various absorption experiments.

Herlach et al. [13] made the first cyclotron resonance measurements in megagauss fields, using a laser as a single wave-length light source. Resonance was observed from the detector signal when the varying magnetic field hit the right value.

The Institute of Solid State Physics of the University of Tokyo has had a very extensive megagauss field program for many years. Some of the more recent work, mainly with laser light sources, is discussed in the article by Franse and Miura [6]. An earlier survey of 
this program is given by Miura et al. [14], while Kido et al. [15] describe their modification of an Image Intensifier into a time-resolved, spectrographic camera.

Finally, we note that very high field, single wave-length, optical experiments are occasionally reported by a group from Russia. Earlier work of this kind is noted as Ref. 23 in the survey by Fowler et al. [12]. Recently, Markevsev et al. [16] used Faraday rotation with laser light sources to study some materials in fields up to $500 \mathrm{~T}$.

\subsection{Electrical Diagnostics}

Most of the electrical measurements made in megagauss field systems are of sample resistance or magnetization, to which this discussion is confined. The large values of $d B / d t$ developed in such systems are of a mixed blessing. As noted earlier, they allow use of quite small induction probes for field measurements. In magnetization measurements, the variable usually measured is $d M / d t$, obtained from the signal developed in a coil that houses the experimental sample. This signal, $(d M / d B) \cdot(d B / d t)$, increases directly with $d B / d t$.

The major difficulty posed by large values of $d B / d t$ is that of avoiding inductive pickup voltage in the sample and leads. This requires making the projected area of the sample and its electrical leads perpendicular to the field as small as possible.

Each electrical measurement in megagauss fields usually requires ingenuity on the part of the experimenter to reduce, or largely eliminate, this voltage. Examples include the use of "contactless" measurements such as those obtained by Sakakibara, Goto, and Miura [17]. Here, thin samples of the superconductor, YBCO, were sandwiched between two small parallel coils, one a transmitter of $20-\mathrm{MHz}$ radiation, the other a receiver. The amplitude of the signal received is a function of the sample conductivity. With this technique, described earlier by Kido et al. [15], the resistivity of YBCO was obtained over 
a wide range of temperature and magnetic fields. Pavlovskii et al. [18] have extended this technique by using microwaves $(8 \mathrm{~mm})$ for the transmitter in their studies of YBCO. Microwave penetration of the sample occurs at that value of the magnetic field when the sample starts to become resistive. Filters may be used to screen out most of the noise arising from $d B / d t$, since this noise is usually of much lower frequency than that of the transmitters. Fowler et al. [19] describe use of a high-band pass filter in measurements of the dHvA effect in YBCO. Strip generators (Fig. 1) were used to create the high fields. Fourier analysis of the $d B / d t$ curve of Fig. 2 shows very little frequency content above $200 \mathrm{kHz}$. The magnetization signal was passed through a high-band pass filter whose cutoff was centered at $500 \mathrm{kHz}$. Simulation of the theoretical magnetization signal showed that it was very little affected - at least at the frequencies reported there.

Several constraints affect these techniques. For the contactless high-frequency technique, the frequency must be high enough that several modulation cycles occur in times where measurable changes occur in the magnetic field pulse. In turn, the sample thickness (for most experiments) should be less than a skin depth thick, but not so thin that anomalous-size effects are significant [20]. Similar considerations apply to direct signal filtering. The filter cutoff frequencies should be high enough to filter out the $d B / d t$ noise but, at the same time, not so high as to remove a significant part of the experimental signal under investigation.

\section{CURRENT ACTIVITIES}

The explosive flux compression facility serves as an adjunct to the Pulsed Field arm of the National High Magnetic Field Laboratory at Los Alamos. Most of the pulsed field work is expected to take place in the long-pulse magnetic facility described by Campbell et al. [21] at this conference. Currently, peak fields here are restricted to around $60 \mathrm{~T}$. The explosive facilities may be used when higher fields are required. 


\subsection{Recent YBCO Measurements}

Kudasov et al. [22] give recent results on the upper critical field of YBCO. Values for polycrystalline platelets are given on their Fig. 2. Data plotted from about $25 \mathrm{~K}$ to $75 \mathrm{~K}$ give a concave upward curve with increasing upward slope at $25 \mathrm{~K}$, where the critical field is about $180 \mathrm{~T}$. A reasonable extrapolation of the data would be compatible with a critical field of several hundred Tesla at, say, $4 \mathrm{~K}$. Their most recent measurements used the microwave technique outlined in Sec. 2.2. Here, 8-mm wavelength radiation was used. The microwave signals were transmitted and received in metal wave guides nearly up to the high magnetic field generator. Dielectric wave guides, connected to the conventional metal wave guides by small copper horns, carried the transmitted and received signals through the high-field systems. When the same technique was used on monocrystalline samples, critical fields of only 75-100 $\mathrm{T}$ were obtained, even with the orientation where $B$ was perpendicular to the $\mathrm{C}$-axis. The authors attributed this anomalous result to poor quality of the sample films.

Recently, resistance measurements have been made on monocrystalline YBCO in megagauss fields. Smith et al. [23] have given a short summary of some of the results while a more extensive discussion by Goettee et al. [24] is in press. Here, the YBCO samples were deposited on [100] $\mathrm{LaAlO}_{3}$ substrates with the C-axis perpendicular to the plane. Photo etching produced meander-line patterns of about $60-\mu \mathrm{m}$ wide and less than $200-\mathrm{nm}$ thick. Resistances were measured with a standard four-terminal technique. Two kinds of high-field systems were used: the single-stage systems of Fig. 1, and for fields greater than $150 \mathrm{~T}$, a two-stage system as described in Ref. [12]. As noted in Ref. [24], from the rather sketchy data when $B$ is perpendicular to the $\mathrm{C}$-axis, it is difficult to estimate the lowtemperature critical field. Various estimates gave an extreme value of nearly $1000 \mathrm{~T}$, and a value of $600 \mathrm{~T}$, which is thought to be more reasonable. Another point noted in Ref. [24] dealt with the quality of the YBCO films. Of the several shots fired in the program, two 
(unreported) samples with visible pinholes, apparently heated during the experiments and showed unusual resistance oscillations after they became resistive. This supports the view of Kudasov et al. [21], expressed above, that poor film quality led to spurious results.

\subsection{Near-Term Experiments}

Almost irnmediately after this conference, a joint Russian/Los Alamos high-field shot series will be carried out at the Los Alamos flux compression facility. The high-field sources to be used are cylindrical explosive flux compressors developed by Pavlovskii et al. [25], a number of which have been purchased. (Los Alamos will supply the explosives required.) Among the benefits to be enjoyed from this exercise is that of cementing a working relationship with colleagues having similar interests, and adapting our facilities to use their sources for a number of possible experiments that might be carried out here, as well as in Russia.

In this first series, however, we hope to shed some light on the low-temperature, upper-critical field of YBCO. We have agreed to use the microwave technique described by Kudasov et al. [22] as discussed above, but to use an even shorter wavelength (4 mm). The experimental volume available, with this smaller wave guide, should allow good measurements in fields greater than $500 \mathrm{~T}$. 
[1] F. Herlach, Prog. in Phys. XXXI, Pt. I, 341 (1968).

[2] H. Knoepfel, "Pulsed High Magnetic Fields," North-Holland Publ. Co., Amsterdam and London (1970).

[3] C. M. Fowler, R. S. Caird, and W. B. Garn, Los Alamos National Laboratory Report, LA-5890-MS (1975).

[4] C. M. Fowler, Los Alamos National Laboratory Report, LA-UR 90-2798, p. 9 (1990).

[5] N. Miura, H. Nojiri, T. Takamasu, T. Goto, K. Uchida, H. A. Katori, T. Haruyama and S. Todo, to be published in: Megagauss Magnetic Field Generation and Pulsed Power Applications," eds. M. Cowan and R. B. Spielman (Nova Science Publishers, New York, in press).

[6] J.J.M. Franse and N. Miura, MRS Bulletin, Vol. XVIII, No. 8, p. 44 (August 1993).

[7] C. M. Fowler, Los Alamos National Laboratory Reports; LA-9956-MS, Part 1, Eqs. (2.15) and (2.17), (1984); LA-9956-MS, Part 2, Eqs. (4.56) and (4.57), (1988).

[8] C. M. Fowler, R. S. Caird, and W. B. Garn, Los Alamos National Report, LA-5890MS (1975) pp. 14-18.

[9] A I. Pavlovskii, N. P. Kolokolchikov, O. M. Tatsenko, A. I. Bykov, M. I. Dolotenko, and A. A. Karpikov, in: Megagauss Physics and Technology, ed. P. J. Turchi (Plenum Press, New York and London, 1980) p. 627.

[10] C. M. Fowler, R. S. Caird, W. B. Garn, D. J. Erickson, and B. L. Freeman, J. Less. Comm. Met. 62 (1978) 397.

[11] W. B. Garn, R. S. Caird, D. B. Thomson, and C. M. Fowler, Rev. Sci. Instr. 37 (1966), 762 . 
[12] C. M. Fowler, R. S. Caird, D. J. Erickson, B. L. Freeman, and W. B. Garn in: Physics in High Magnetic Fields, ed. S. Chikazumi and N. Miura (Springer-Verlag, Berlin, Heidelberg, New York 1981) p. 54.

[13] F. Herlach, J. Davis, R. Schmidt, and H. Spector, Phys. Rev. B 10 (1974), 682.

[14] N. Miura, T. Goto, K. Nakao, S. Takeyama, and T. Sakakibara in: Megagauss Technology and Pulsed Power Applications, eds. C. M. Fowler, R. S. Caird, and D. J. Erickson (Plenum Press, New York, 1987) p. 209.

[15] G. Kido, N. Miura, M. Akishiro, H. Katayama, and S. Chikazumi in: Physics in High Magnetic Fields, eds. S. Chikazumi and N. Miura, Springer-Verlag, Berlin, Heidelberg; New York, 1981) p. 72.

[16] I. M. Markevsev, A. I. Pavlovskii, V. V. Platonov, D. V. Sosnin, O. M. Tatsenko, V. V. Druzhinin, A. S. Lagutin, P. Nicitin, and A. I. Sawshuk, to be published in: Megagauss Magnetic Field Generation and Pulsed Power Applications, eds. M. Cowan and R. B. Spielman (Nova Science Publishers, New York, in press).

[17] T. Sakakibara, T. Goto, and N. Miura, Rev. Sci. Instrum. 60 (3) (1989), 444.

[18] A. I. Pavlovskii, O. M. Tatsenko, V. V. Platonov, S. A. Voronov, N. P. Kolokolchikov, I. M. Markevtsev, Yu. N. Deryugin, V. V. Druzhinin, and Yu. P. Shcherbak, in: Megagauss Fields and Pulsed Power Systems, Eds. V. M. Titov and G. A. Shvetsov (Nova Scien'ce Publishers, New York, 1990), 139.

[19] C. M. Fowler, B. L. Freeman, W. L. Hults, J. C. King, F. M. Mueller, and J. W. Smith, Phys. Rev. Lett. 68, No. 4 (1992), 534. 
[20] In som€ recent copper magnetoresistance experiments at Los Alamos, size effects set a lower limit to the thickness of the samples that could be used. This subject is discussed by F. R. Fickett in the report, "INCRA Project No. 186, A Preliminary Investigation of the Behavior of High Purity Copper in High Magnetic Fields," National Bureau of Standards and Technology, Boulder, CO, USA.

[21] L. J. Campbell, J. Crow, D. M. Parkin, H. J. Schneider-Muntau, and N. Sullivan, to be published in the proceedings of this conference.

[22] Yu. B. Kudasov, A. I. Pavlovskii, V. V. Platonov, and O. M. Tatsenko, to be published in, Megagauss Magnetic Field Generation and Pulsed Power Applications, Eds. M. Cowan and R. B. Spielman (Nova Science Publishers, New York), in press.

[23] J. L. Smith, C. M. Fowler, B. L. Freeman, W. L. Hults, J. C. King, F. M. Mueller, D. G. Rickel, J. S. Brooks, J. D. Goettee, P. M. Mankiewich, E. I. De Obaldia, W. J. Skocpol, and M. L. O'Malley, Physica B 186-188 (1993), 104.

[24] J. D. Goettee, J. S. Brooks, D. G. Rickel, B. L. Freeman, C. M. Fowler, J. C. King, P. M. Mankiewich, W. J. Skocpol, E. I. De Obaldia, M. L. O'Malley, and J. L. Smith, to be published in: Megagauss Field Ceneration and Pulsed. Power Applications, Eds. M. Cowan and R. B. Spielman (Nova Science Publishers, New York), in press. 
Fig. 1. Schematic drawing of the 1-MG system showing a load coil and metal conductors overlaid with sheet explosive. The position of the conductors is shown by dotted lines when the explosive detonation fronts have moved to the position $D$.

Fig. 2. Curves of magnetic field and its time derivative vs time generated by a 1-MG system with a load coil of $12.7-\mathrm{mm}$ diameter and $76.2-\mathrm{mm}$ long.

Fig. 3. Circuit schematic of a flux compression generator $L(t)$ powering a load $L_{1}$. Inductive and resistive loss terms are given by $\ell$ and $R$. 


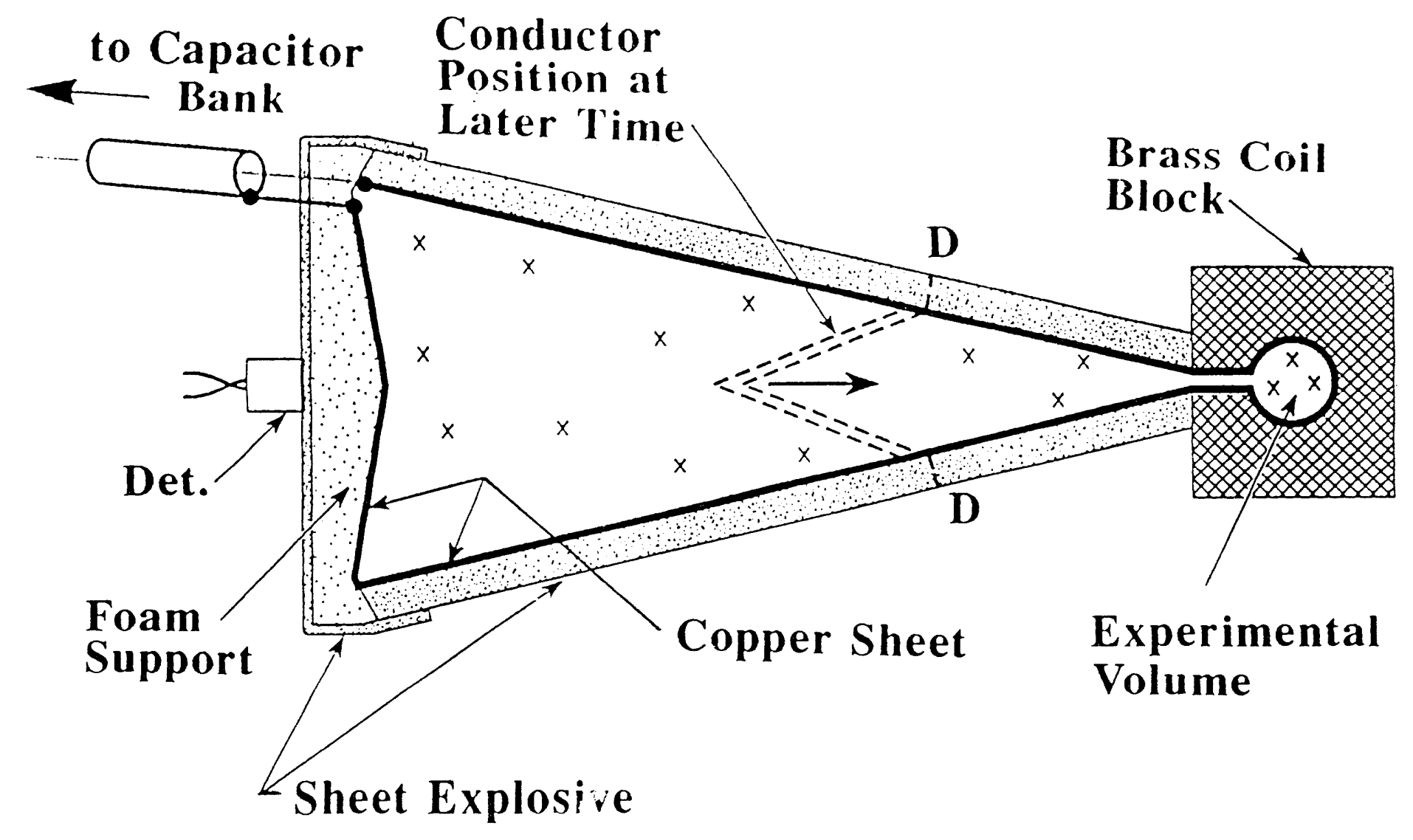




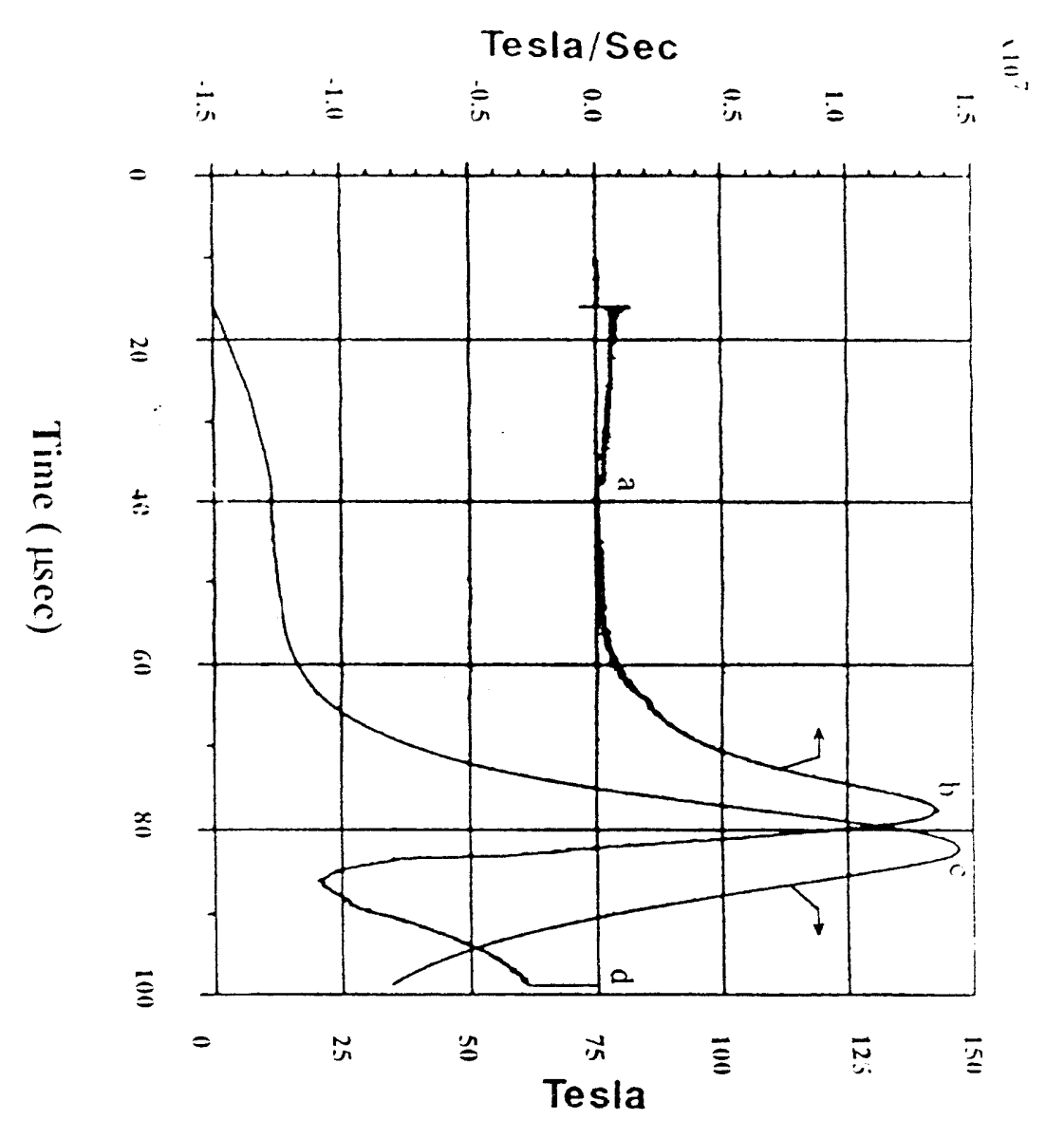




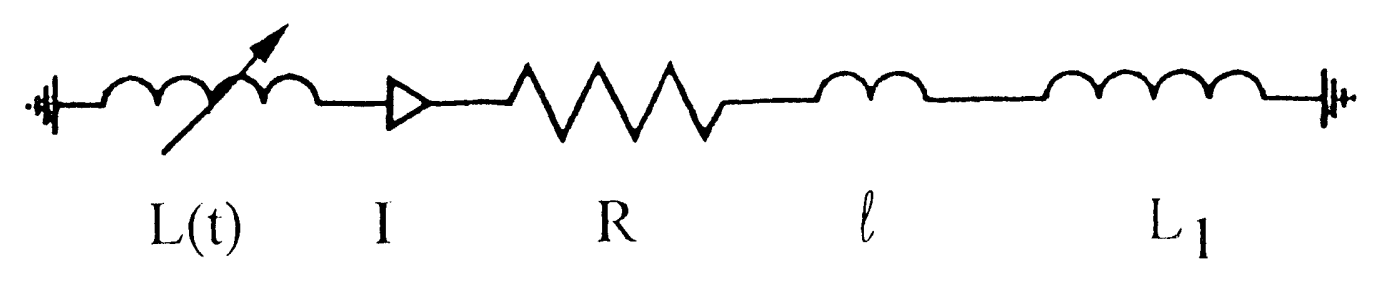




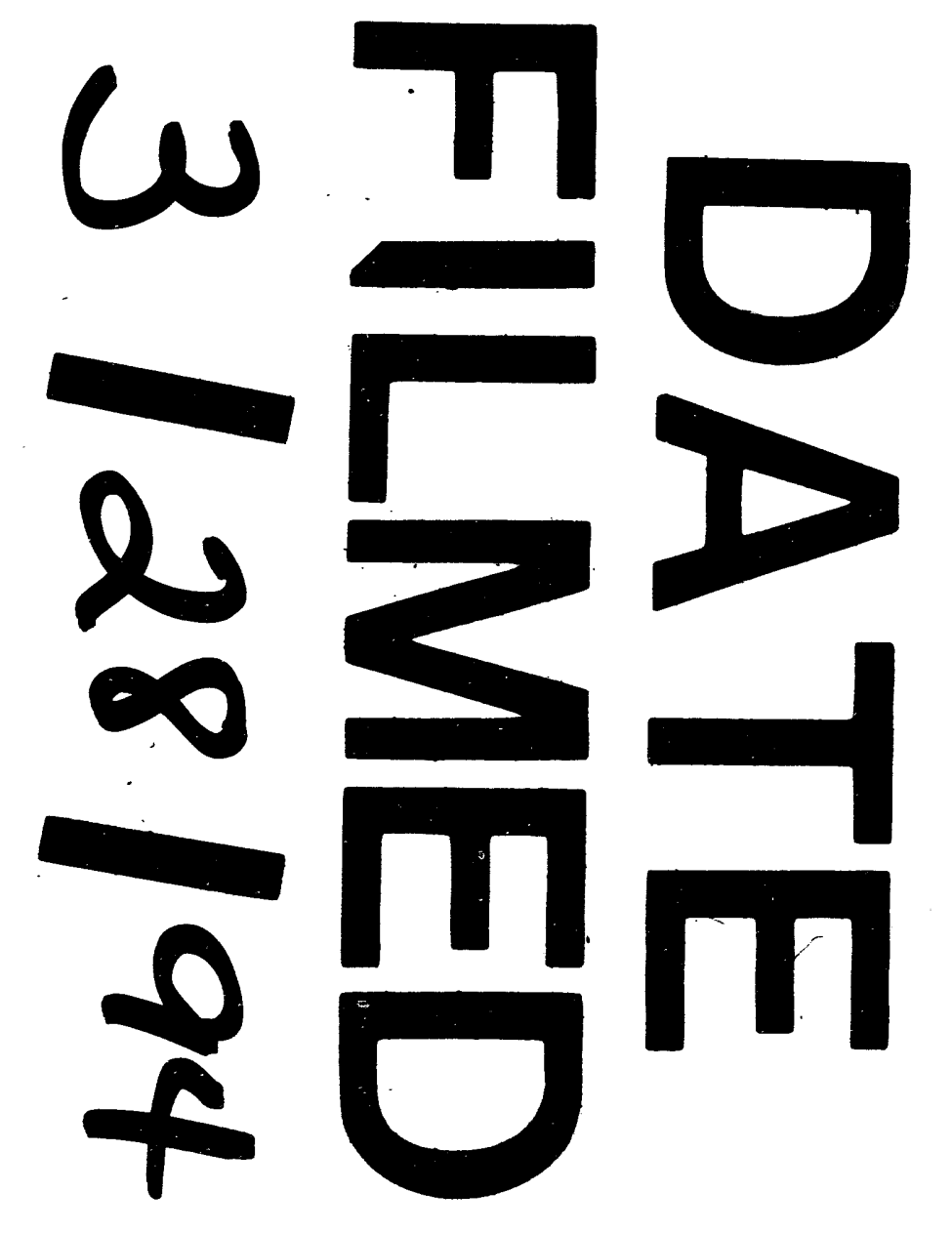



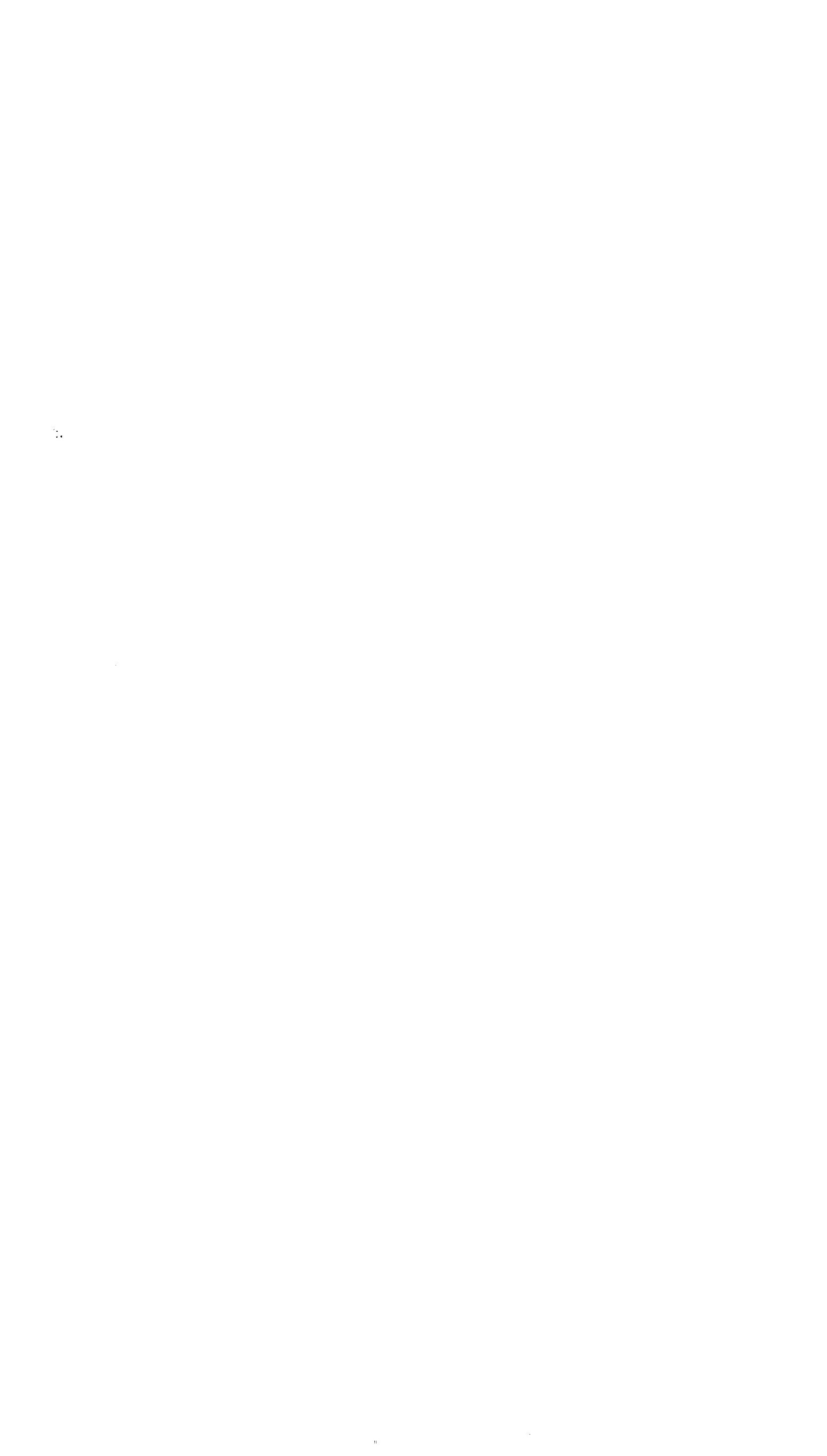